\title{
THE LOCATION OF NON-THERMAL VELOCITY IN THE EARLY PHASES OF LARGE FLARES-REVEALING PRE-ERUPTION FLUX ROPES
}

\author{
Louise K. Harra $^{1}$, Sarah Matthews ${ }^{1}$, J. L. Culhane ${ }^{1}$, Mark C. M. Cheung ${ }^{2}$, Eduard P. Kontar ${ }^{3}$, and Hirohisa Hara ${ }^{4}$ \\ ${ }^{1}$ UCL-Mullard Space Science Laboratory, Holmbury St Mary, Dorking, Surrey RH5 6NT, UK; 1.harra@ ucl.ac.uk \\ ${ }^{2}$ Lockheed Martin Solar and Astrophysics Laboratory, 3251 Hanover Street, Building/252, Palo Alto, CA 94304, USA \\ ${ }^{3}$ School of Physics and Astronomy, University of Glasgow, Glasgow G12 8QQ, UK \\ ${ }^{4}$ National Astronomical Observatory of Japan, Osawa, Mitaka, Tokyo 181-8588, Japan \\ Received 2013 April 12; accepted 2013 July 25; published 2013 August 23
}

\begin{abstract}
Non-thermal velocity measurements of the solar atmosphere, particularly from UV and X-ray emission lines have demonstrated over the decades that this parameter is important in understanding the triggering of solar flares. Enhancements have often been observed before intensity enhancements are seen. However, until the launch of Hinode, it has been difficult to determine the spatial location of the enhancements to better understand the source region. The Hinode EUV Imaging Spectrometer has the spectral and spatial resolution to allow us to probe the early stages of flares in detail. We analyze four events, all of which are GOES M- or X-classification flares, and all are located toward the limb for ease of flare geometry interpretation. Three of the flares were eruptive and one was confined. In all events, pre-flare enhancement in non-thermal velocity at the base of the active region and its surroundings has been found. These enhancements seem to be consistent with the footpoints of the dimming regions, and hence may be highlighting the activation of a coronal flux rope for the three eruptive events. In addition, pre-flare enhancements in non-thermal velocity were found above the looptops for the three eruptive events.
\end{abstract}

Key word: Sun: flares

Online-only material: animations, color figures

\section{INTRODUCTION}

Signatures that indicate reliably in advance if a flare or a coronal mass ejection (CME) will occur have been sought after for decades. These signatures have been wide-ranging and include the emergence of new flux (e.g., Kurokawa et al. 2002) and the complexity and shear within the magnetic field. A good example is the complex active region that produced a GOES $\mathrm{X}$-class flare in 2006 December. It showed new emerging flux which developed complex twist before the flares and CMEs. Inoue et al. (2012) analyzed the three-dimensional structure of the magnetic field in this active region. They found that the central region near the polarity inversion line contained a mix of oppositely twisted magnetic fields and strong currents which are likely to play a prominent role in the onset of the large flare. In the same flare, spectroscopic observations revealed antisymmetric Doppler shifts along the filament length that occurred before the impulsive phase (Williams et al. 2009). These are consistent with the expansion of a twisted flux rope due to the magnetohydrodynamic (MHD) helical kink instability. This occurred several minutes before the flare onset.

In the corona it has been found that non-thermal velocities (excess line width in emission lines) peak early in flares with a magnitude of approximately $200 \mathrm{~km} \mathrm{~s}^{-1}$ (Doschek et al. 1980). These early signatures have been found in flares with a wide range of energies, and the signature has been seen tens of minutes before a flare peak (Harra et al. 2001). Such an early response to the flaring suggested that this parameter is important in the early trigger phase of flares. However its location has been difficult to determine due to limited or no spatial resolution in spectrometers before the launch of Hinode. Analysis of imaging data (both hard X-ray, HXR and EUV) by Warren \& Warshall (2001) found that pre-flare brightenings occurred in a different location to the flare footpoints as measured in
HXR. This suggests that the pre-flare energy release is occurring in different loops to those subsequently involved in the flare. Recent observations from Hinode tracking the non-thermal velocity before the X-class flare on the 2006 December 13 have shown that this parameter can start to rise many hours before the flare occurs (Harra et al. 2009). Indeed, the increase occurs after the saturation of the injected helicity measured by Magara \& Tsuneta (2008). This flare was one of the largest observed by Hinode during the first few years of the mission. However, even in small B-class flares, this increase in non-thermal velocity has been seen around $1 \mathrm{hr}$ before the flare began (Wallace et al. 2010). This occurred at the location between new emerging flux and pre-existing flux. Another example of loop-loop interaction was analyzed by Watanabe et al. (2012). This was a larger GOES C9.7 flare, and a bi-directional flow was observed in the He II emission line around nine minutes before the flare occurred.

The determination of the source of enhanced non-thermal velocity has been carried out for the impulsive phase of flares. Hara et al. (2011) carried out a detailed analysis of a flare at disk center that showed hot sources in Fe XXIII and Fe XXIV with excess line broadening. A study of the geometrical relationship of the different phenomena observed concluded that these were signatures of magnetic reconnection at the looptop. Another flare studied by Milligan (2011) showed that the non-thermal velocities at the footpoints during the impulsive phase have a strong correlation with Doppler velocity, and hence may be due to a superposition of flows as a result of chromospheric evaporation caused by non-thermal electrons. At the same time, the non-thermal broadening observed in the quiet solar corona (e.g., Hassler et al. 1990) is interpreted as an indication of MHD waves. While MHD turbulence is expected to be an essential ingredient in many particle acceleration and magnetic reconnection scenarios, the observational evidence is very limited (e.g., Kontar et al. 2011). Therefore, the non-thermal 


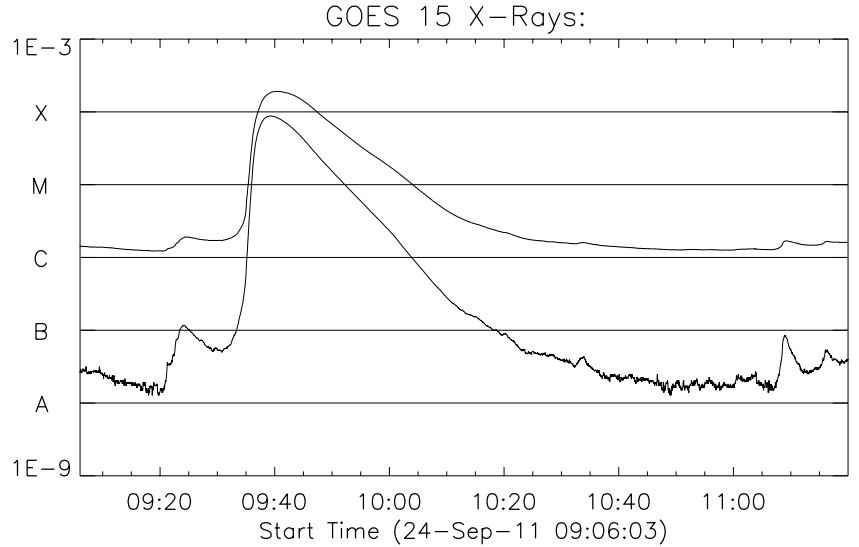

GOES 15 X-Rays:

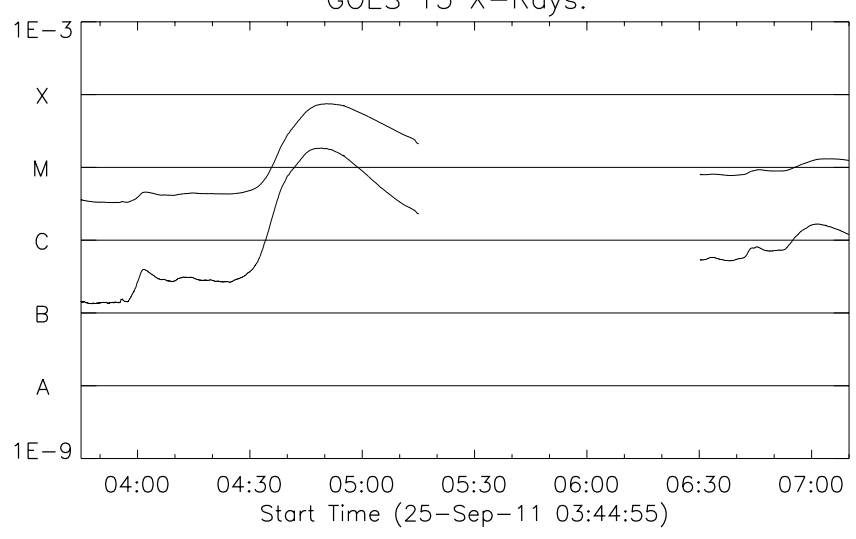

GOES 15 X-Rays:

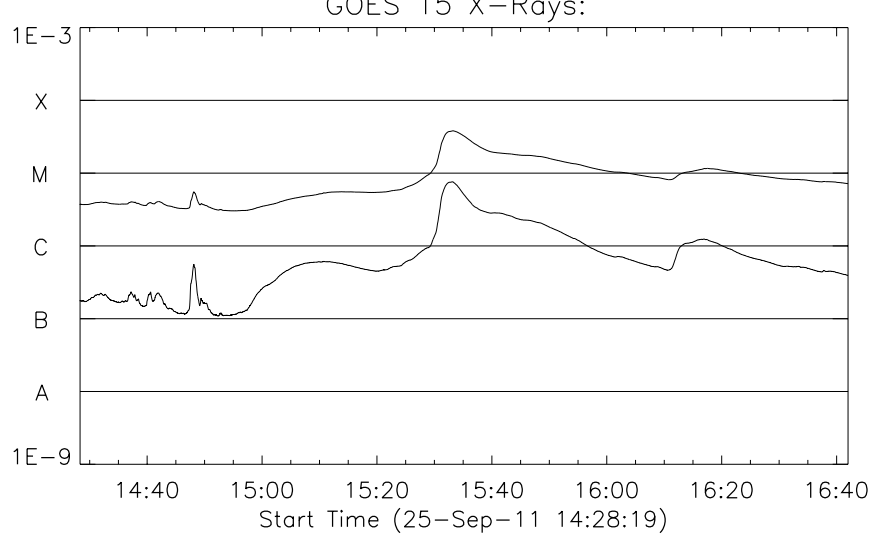

GOES 15 X-Rays:

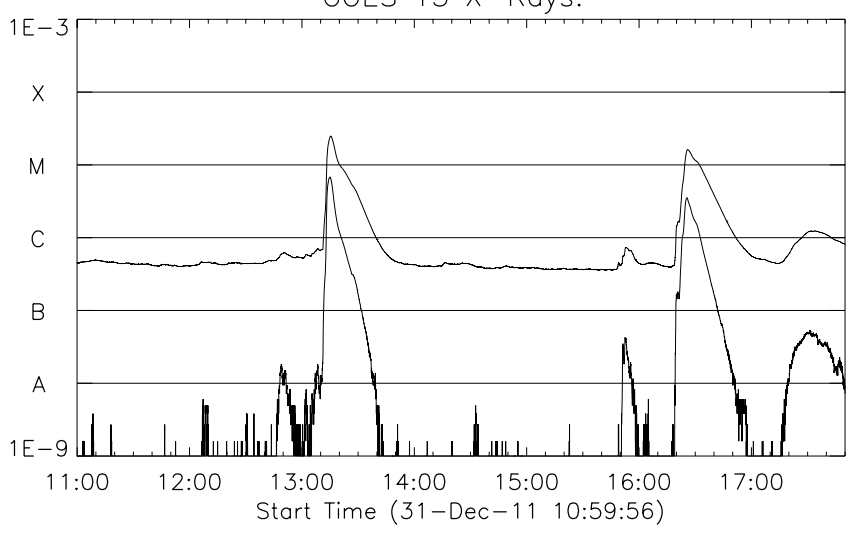

Figure 1. GOES light curves for each for the four flares studied.
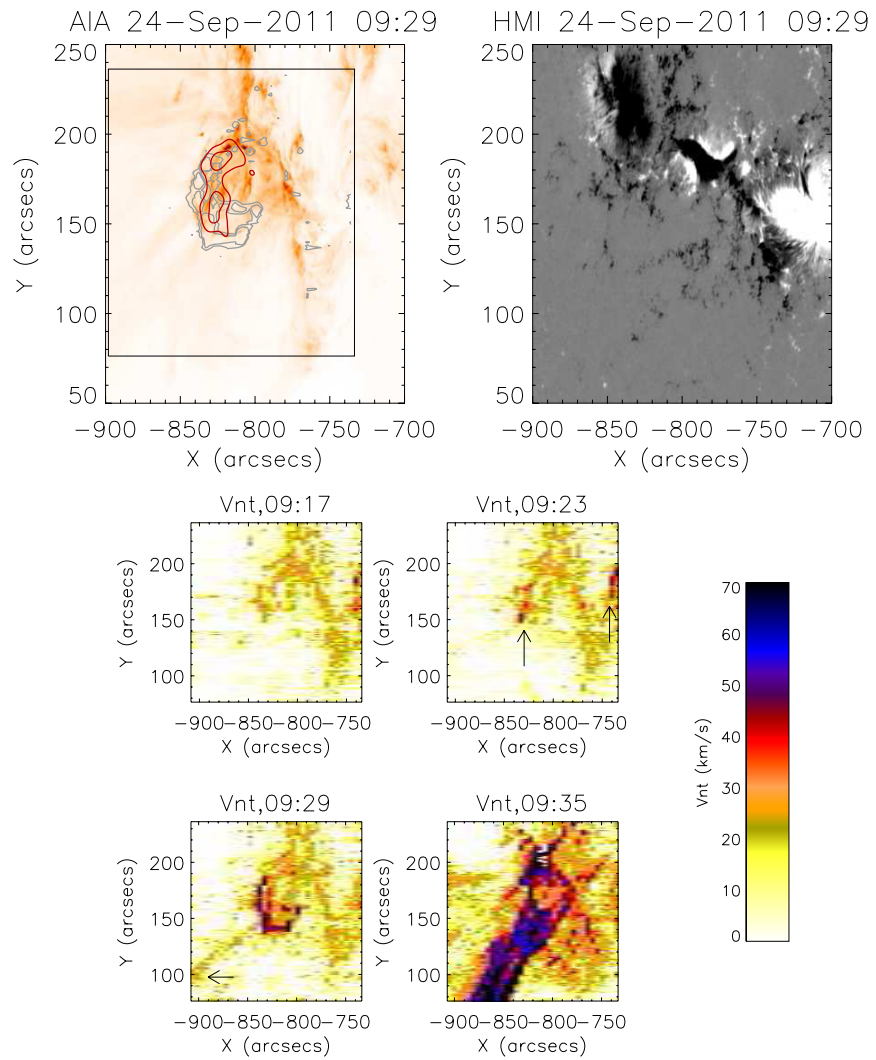

Figure 2. Top left image shows an AIA 193 image at 09:29 with non-thermal velocity (Vnt) contours (Fe XII, $30-40 \mathrm{~km} \mathrm{~s}^{-1}$ ) in gray and the RHESSI contours in red (3-6 keV). The box shows the location of the EIS field of view. The top right panel shows the line-of-sight magnetic field data from HMI. Below shows four views taken at different times of Vnt images derived from Fe XII showing the location of the sources of high Vnt. The image at 09:17UT shows the Vnt of the active region before flare related changes occur. In the image at 09:23 UT there are a few pixels showing enhancements - this is before the event starts. An arrow highlights these. In the frame at 09:29, an arrow highlights the early phases of the eruption.

(A color version of this figure is available in the online journal.)

velocity locations could reveal the locations of magnetic reconnection, enhanced plasma turbulence and/or flows, all critical, but poorly observed, ingredients of solar flare model.

In this work, we concentrate on analyzing four large flares that are located close to the limb, and have data from Hinode, the Solar Dynamics Observatory (SDO), GOES, and RHESSI. Our aim was to determine spatially where the pre-flare signature in the non-thermal velocity is located, to try to determine the physical origin.

\section{DATA ANALYSIS}

We analyzed data for four GOES M- or X-class flares observed simultaneously by the Hinode (Kosugi et al. 2007) and RHESSI (Lin et al. 2002) spacecraft and concentrated on the data from the EUV Imaging Spectrometer (EIS; Culhane et al. 2007). The observations used $5^{\prime \prime}$ steps in the $x$-direction to build up an image which is $180^{\prime \prime} \times 160^{\prime \prime}$ in just under five minutes. The study name for this is HH_Flare_180x160_v2. The standard calibration was used through the routine eis_prep, and the slit tilt and the orbital variation of line position were corrected for. We concentrated on the strong coronal Fe XII $\lambda 195$ emission line and for each pixel fitted the line using a single Gaussian profile. This results in a line width and intensity "image" for each raster scan. From this the non-thermal velocity can be derived by subtracting the thermal width and the instrumental 

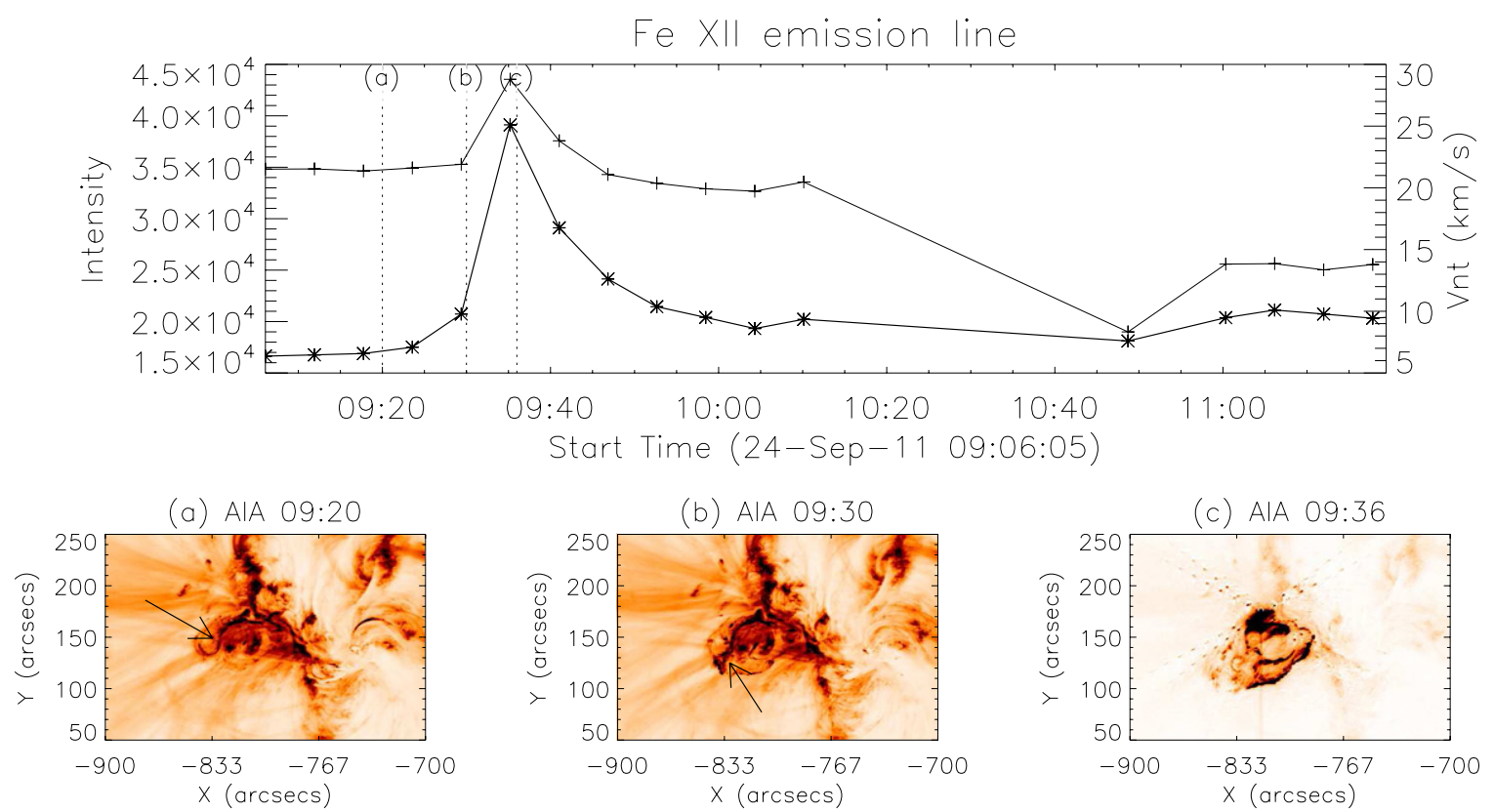

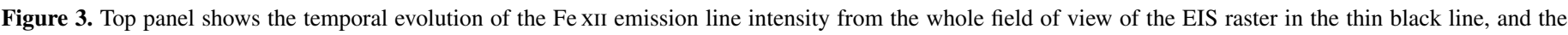

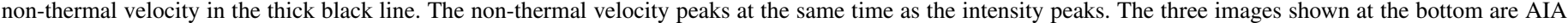

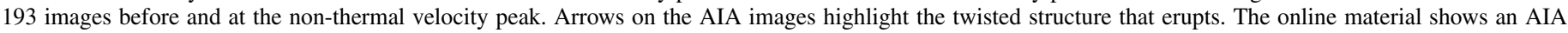
animation combining the 171, the 335, and the 94 wave bands for this flare. An intensity light curve of each waveband is also shown.

(An animation and color version of this figure are available in the online journal.)

width. We made use of the Solarsoft routine eis_width2velocity within the EIS software tree. This uses the equation

$$
\mathrm{FWHM}^{2}=\left(\operatorname{instr}_{\mathrm{fwhm}}\right)^{2}+4 \ln (2)(\lambda / c)^{2}\left(v_{t}^{2}+\left(v_{\mathrm{nt}}\right)^{2}\right),
$$

where $v_{t}$ is the thermal velocity, FWHM is the full width at halfmaximum of each line profile, $\lambda$ is the wavelength of the peak of the emission line, $c$ is the speed of light, $v_{\text {nt }}$ is the non-thermal velocity, and instr $f_{\text {fwhm }}$ is the instrumental width.

In addition we analyzed data from the Atmospheric Imaging Assembly (AIA; Lemen et al. 2012) on board SDO. In order to aid the alignment between AIA and EIS, we used the 195 $\AA$ emission line in both instruments and manually aligned the two data sets by means of feature recognition. This line is a particularly strong and well-resolved line and is sensitive to the plasma temperatures of $\approx 1.2 \mathrm{MK}$. During flares, the diffraction pattern of the AIA telescope is particularly pronounced and produces a crisscross pattern (resulting from the entrance filter mesh) in AIA images. To mitigate this effect, we deconvolved AIA $193 \AA$ images with the semi-empirical point-spread function detailed in Grigis et al. (2013, available from the Solarsoft AIA package) using iterative Richardson-Lucy (RL) deconvolution (Richardson 1972; Lucy 1974). Since RL deconvolution preserves the sum of pixel values over the image (we deconvolved using full-disk images), it is unable to recover signals in saturated pixels. Nevertheless, application of RL deconvolution substantially mitigates the diffraction patterns and improves image contrast.

We also used the $S D O$ Helioseismic and Magnetic Imager (HMI; Scherrer et al. 2012) line-of-sight magnetograms.

Full-disk EUV images at the $195 \AA$ passband taken by the Extreme UltraViolet Imager (EUVI; Wuelser et al. 2004) on board the STEREO spacecraft. The STEREO behind spacecraft data were used. Base difference images were produced to search for the dimming regions.
The CLEAN algorithm was used to produce HXR images of the flare from RHESSI (Lin et al. 2002) data in the 3-25 keV range with approximately one-minute cadence.

\section{OBSERVATIONS}

We chose the four events to be large in GOES X-ray classification, toward the solar limb to allow more clarity of potential above-the-loop sources. The GOES lightcurves for the four flares are shown in Figure 1.

\subsection{September 24 09:21 Flare}

This flare was designated X1.9 in the GOES X-ray classification, and began at 09:21 UT on 2011 September 24. The flare occurred in NOAA active region 1302, which was at this stage close to the east limb at N12 E60. This region had produced large flares before this one-the previous one being an M1.9 flare peaking at 23:56 UT on the 2011 September 23. The GOES background level stays at a low C-class level for approximately $9 \mathrm{hr}$ before the X1.9 flare occurs. Figure 2 illustrates the location of the Fe XII non-thermal velocity and the RHESSI contours on the AIA $193 \AA$ image. Both the HXR and the non-thermal velocity lie along part of the loop structure seen. However, there is enhanced non-thermal velocity above the loop source-this also lies higher than the HXR source. Figure 2 also shows the non-thermal velocity image at four times-the first showing the non-thermal velocity in the active region before changes related to the flare occur, just before the non-thermal velocity starts to rise, as it rises, and at the peak. At the early phases (09:23 UT raster) there are a few pixels that are enhanced, reaching non-thermal velocities of close to $50 \mathrm{~km} \mathrm{~s}^{-1}$. These occur at the top of the structure that will erupt and on the west side of the flaring region. There are small changes in these pixels from at least 09:06 UT. The eruption starts at 09:35 UT (seen in the AIA animations). Evidence of 

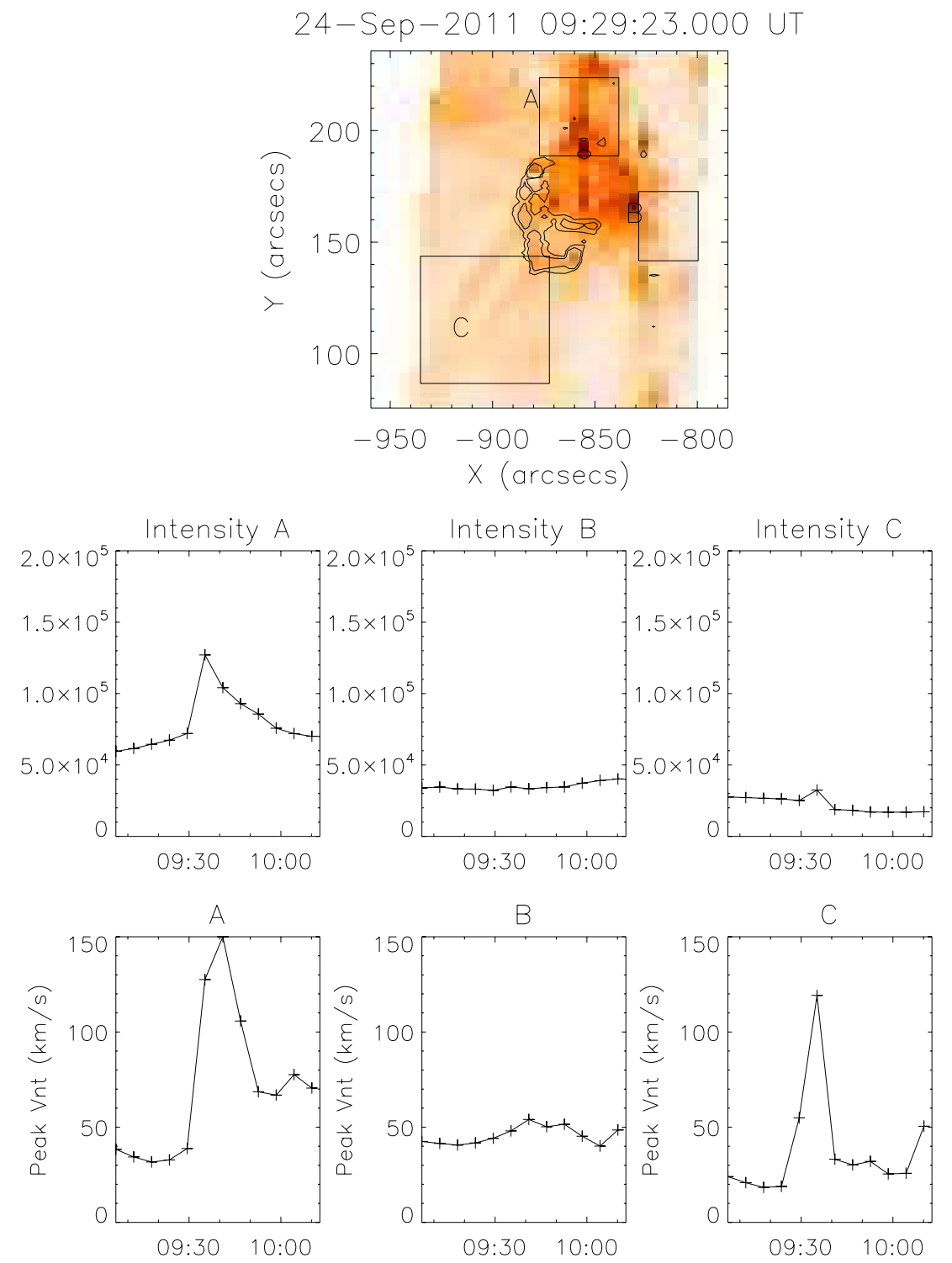

Figure 4. Top image show the EIS intensity of the Fe XII emission line at 09:29. Superimposed are three regions of interest. The contours are the non-thermal velocity contours at levels 30 and $40 \mathrm{~km} \mathrm{~s}^{-1}$. The plots below the image show the intensity in these three regions and at the bottom the peak Vnt in each region. Region A shows the most dramatic changes with the peak in Vnt occurring at the same time as the peak in the intensity. Region C also shows significant changes in Vnt.

(A color version of this figure is available in the online journal.)

the early phases of this are seen in the non-thermal velocity image starting at 09:29 UT. In this image there are enhanced non-thermal velocities seen around $20 \mathrm{~km} \mathrm{~s}^{-1}$ which lie in the trajectory path of the eruption (at the top of the loop shape and toward the southeast direction marked by an arrow). The final non-thermal velocity image depicted (at 09:35 UT) shows a long stream of material which is the eruption in full flow. In AIA data the eruption stops around 09:44 UT. The LASCO CME catalog (Yashiro et al. 2004) shows a partial halo with a very fast linear speed of $1936 \mathrm{~km} \mathrm{~s}^{-1}$.

Figure 3 shows the lightcurves of the Fe XII intensity and nonthermal velocity along with images from AIA $193 \AA$ before, at, and after the non-thermal velocity peak. In this example, within the EIS time resolution, the intensity and non-thermal velocity peak at the same time. At 09:20 UT a clearly twisted filamentary structure is visible (highlighted by an arrow). In the next time frame shown at 09:30 UT this structure is starting to rise and untwist. At this stage the non-thermal velocity has started to rise. In the final frame both intensity and non-thermal velocity are at their peak, and the flare loops have started to form.

Figure 4 shows lightcurves of the EIS intensity and nonthermal velocity for small regions. The highest intensity is seen from the region that has the twisted structure seen in AIA (region A). In this region the intensity is so strong that some of the pixels are saturated, making it difficult to measure accurately the line width or intensity. Taking this into consideration, the intensity and non-thermal velocity are peaking closely in time as far as we can measure. Region B shows no significant enhancements in the EIS intensity, but the non-thermal velocity here is enhanced to close to $50 \mathrm{~km} \mathrm{~s}^{-1}$ during the time shown, and studying the spatial changes, this region is showing pixel enhancements for tens of minutes before the flare. What is interesting is that this region is not involved directly in the main flare process itself and is off to one side of the main flare core. In this region there is no detectable HXR emission. Region C lies above the erupting structure. There is a sharp rise in non-thermal velocity 

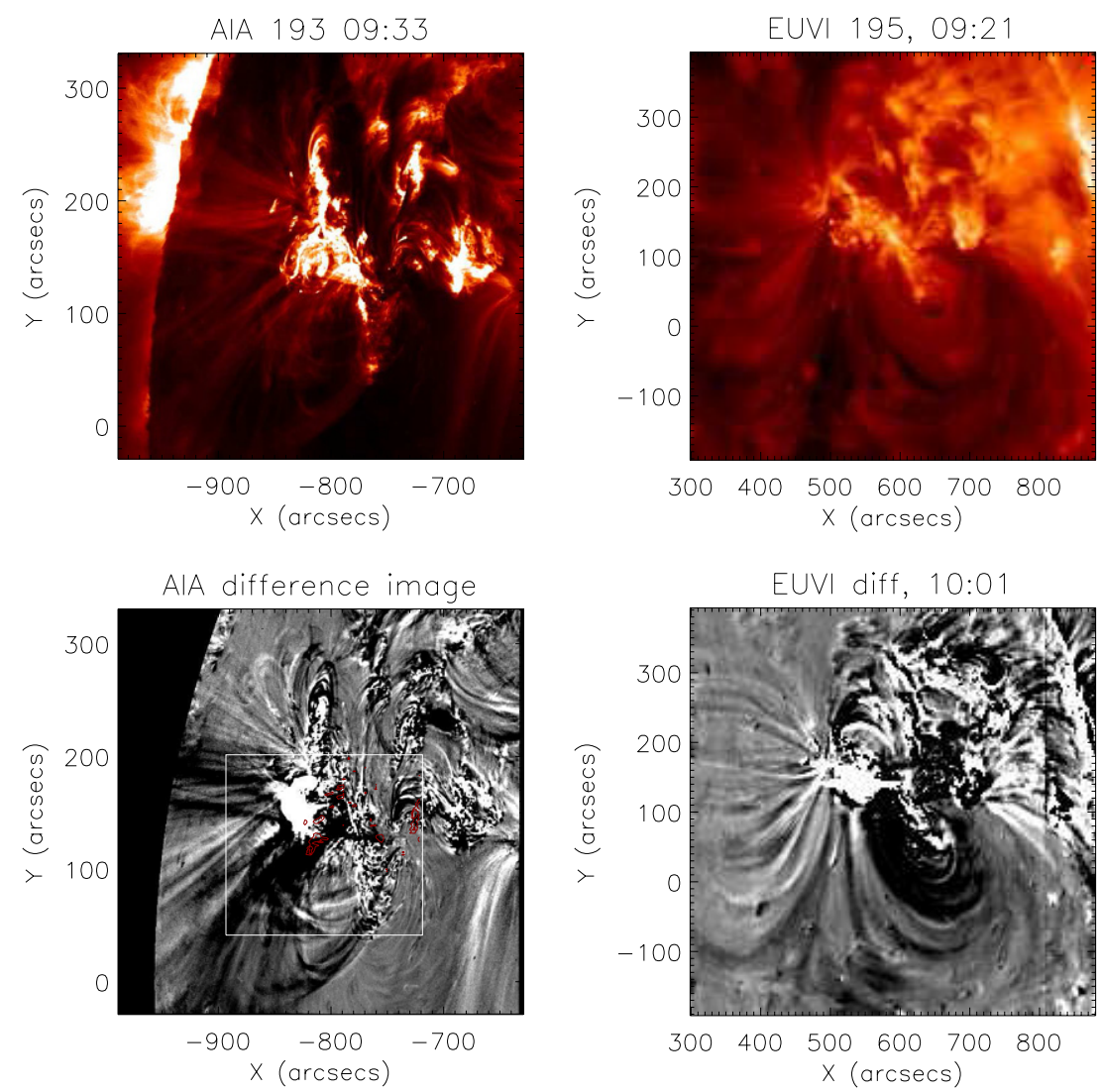

Figure 5. Top left panel shows the AIA image before the flare began. The lower left image shows the AIA base difference image which illustrates the dimming regions. The non-thermal velocity contours are shown in red (contour levels 40 and $50 \mathrm{~km} \mathrm{~s}^{-1}$ ) in the pre-flare phase. There are contours at the bottom of where the flare occurred (shown as white) and in addition to the west of the active region close to where the footpoints of the dimming region are (shown as dark). To the west of the active region and at the end of the dimming footpoints there are also enhancements in the non-thermal velocity. The top right image shows the active region as viewed by STEREO B, before the flare occurred at September 24 09:21. The bottom left image shows the base difference image clearly shows the dimming region.

(A color version of this figure is available in the online journal.)

at 09:29 UT before a small change in intensity is seen. This raster covers the time during which the eruption begins. The peak in non-thermal velocity occurs co-temporally with the intensity change. This region has weak intensity but strong "turbulence" in the plasma.

Figure 5 illustrates the AIA image in $193 \AA$ with the difference image below. The non-thermal velocity contours before the eruption are overlaid on the difference image. The non-thermal velocity enhancements occur in the flaring region as well as toward the end of the footpoint of the dimming region. The STEREO-B EUVI data in $195 \AA$ A is also shown with the difference image. Large-scale dimmings are seen which are rooted in the flaring region and to the west of the active region. It is clear that the small region to the west of the active region where we see little intensity change, but enhanced non-thermal velocities, is involved in this dimming region and is important in understanding the full erupting structure.

\subsection{September 25 04:31 UT Flare}

Since the X-class flare described in the previous subsection, six M-class flares occurred in active region NOAA 1302 . We now look at the seventh M-class flare. It has a GOES classification M7.4, with the flare starting at 04:31 UT. Figure 6 shows an AIA $193 \AA$ image with the EIS Fe XII non-thermal velocity and the HXR contours. The active region has, not surprisingly, changed quite significantly in its magnetic morphology since the $\mathrm{X}$-flare on the September 24. Many more loops are clearly seen by this stage. The largest non-thermal velocities lie along the footpoint regions. There are two HXR sources in this case which both lie close to the footpoint regions. In addition, there is also a region of enhanced non-thermal velocity lying high in the corona above very faint loops. There is no evidence of HXR emission here. Figure 6 also shows the time evolution of the source of the non-thermal velocity - showing the active region before any changes occur, changes as the event is triggered, as the flare starts, and the peak of the event. At the early phase there are enhanced non-thermal velocity pixels in the footpoint regions. The enhanced pixels cover a much larger area than the X-flare on the September 24 in the same active region. In addition, there is a non-thermal velocity enhancement above the loops with no intensity enhancement. As the flare progresses the nonthermal velocity enhancement spreads, outlining a loop-type structure.

Figure 7 shows the temporal evolution of the EIS Fe XII intensity and non-thermal velocity averaged over the whole EIS raster. In this case, within the time cadence of the EIS rasters, the intensity and non-thermal velocity peak at the same time. The non-thermal velocity starts to rise at least 10 minutes before the intensity starts to rise. The AIA images are shown at three times (the start, rise, and peak of the flare). The first eruption seen in the AIA animation starts at 04:33 UT with a rapid expansion of loops. Further ejections occur at 04:56 UT toward the peak of the flare. This may account for the unusual rise phase of the non-thermal velocity, which has an initial enhancement peaking at 04:43 UT and then the second peak 

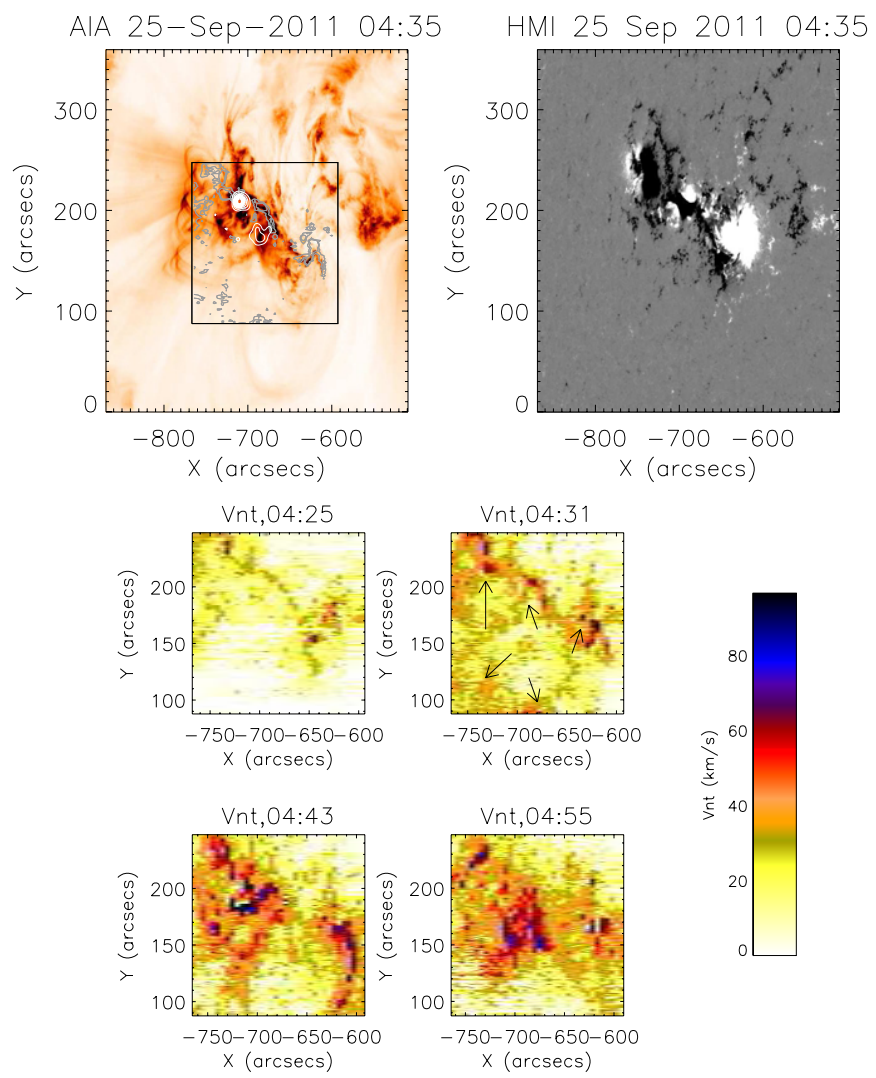

Figure 6. Top left panel shows an AIA 193 image at 04:35 with Vnt contours (Fe XII, 40-50 km s${ }^{-1}$ ) in gray and the RHESSI contours in white (12-25 keV). The box shows the location of the EIS field of view. The top right panel shows the line-of-sight magnetic field data from HMI. Below are four Vnt images derived from Fe XII and taken at different times showing the location of the sources of high Vnt. Arrows highlight the enhancements seen in non-thermal velocity before the flare starts.

(A color version of this figure is available in the online journal.) at 04:54 UT. A partial halo CME is detected by LASCO with a linear speed of $788 \mathrm{~km} \mathrm{~s}^{-1}$.

Figure 8 shows light curves for Fe XII intensity and nonthermal velocity for three regions in the EIS field of view. The highest intensity occurs in the main part of the active region (region $\mathrm{A}$ is a subset of this). The non-thermal velocity of region $\mathrm{A}$ has two distinct peaks. The first one occurs nearly 20 minutes before the intensity peak. The timing of this peak is consistent with the first eruption. Region B shows virtually no intensity increase but there is an enhancement in the nonthermal velocity which again happens tens of minutes before the flare begins. This region has weak intensity, but the non-thermal velocity is showing enhancements well before the flare begins, fluctuating around $50 \mathrm{~km} \mathrm{~s}^{-1}$ for hours (which is above quiescent active region levels), and then jumps to over $70 \mathrm{~km} \mathrm{~s}^{-1}$ as the eruption begins. Region $\mathrm{C}$ above the loops shows two peaks, the first occurring early in the eruption phase, and the second peak at the time of the intensity peak. By this point, post-flare loops have formed, but we are observing a non-thermal velocity enhancement above these.

Figure 9 shows the AIA $193 \AA$ image and difference image below with the non-thermal velocity contours overlaid. The nonthermal velocity enhancements before the eruptive occur over an extended area which appear to lie along the extent of the dimming structure. STEREO-B EUVI data is shown before the eruption with a difference image shown below. The dimming region is in approximately the same location as for the previous flare described on the September 24.

\subsection{September 25 15:26 UT Flare}

Since the M7.4 flare on September 25 04:37 UT there were two more M-class flares and one C-class flare. There was a $4 \mathrm{hr}$ period of relative quiet before the event at 15:26 UT occurred. This event is an M3.7 GOES classification flare that starts at 15:26 UT. Figure 10 shows an AIA $193 \AA$ Amage at 15:32 UT
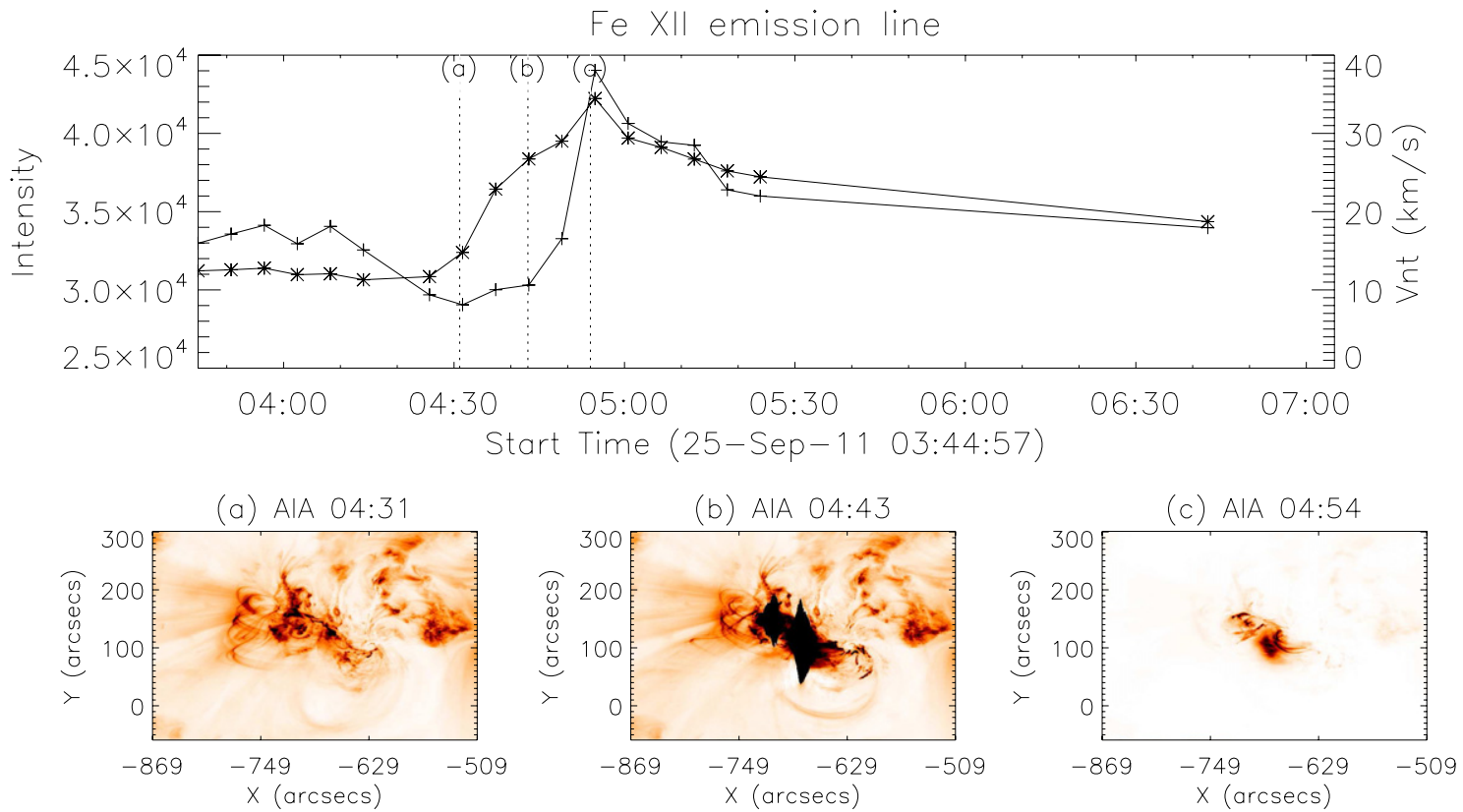

Figure 7. Top plot shows the intensity of the Fe XII emission line from the whole field of view of the EIS raster in the thin black line, and the non-thermal velocity in the thick black line. The non-thermal velocity peaks close in time to the intensity peaks. The three images shown at the bottom are AIA 193 images around the time of the non-thermal velocity peak. The online material shows an AIA animation combining the 171, the 335, and the 94 wave bands for this flare. An intensity light curve of each waveband is also shown.

(An animation and color version of this figure are available in the online journal.) 


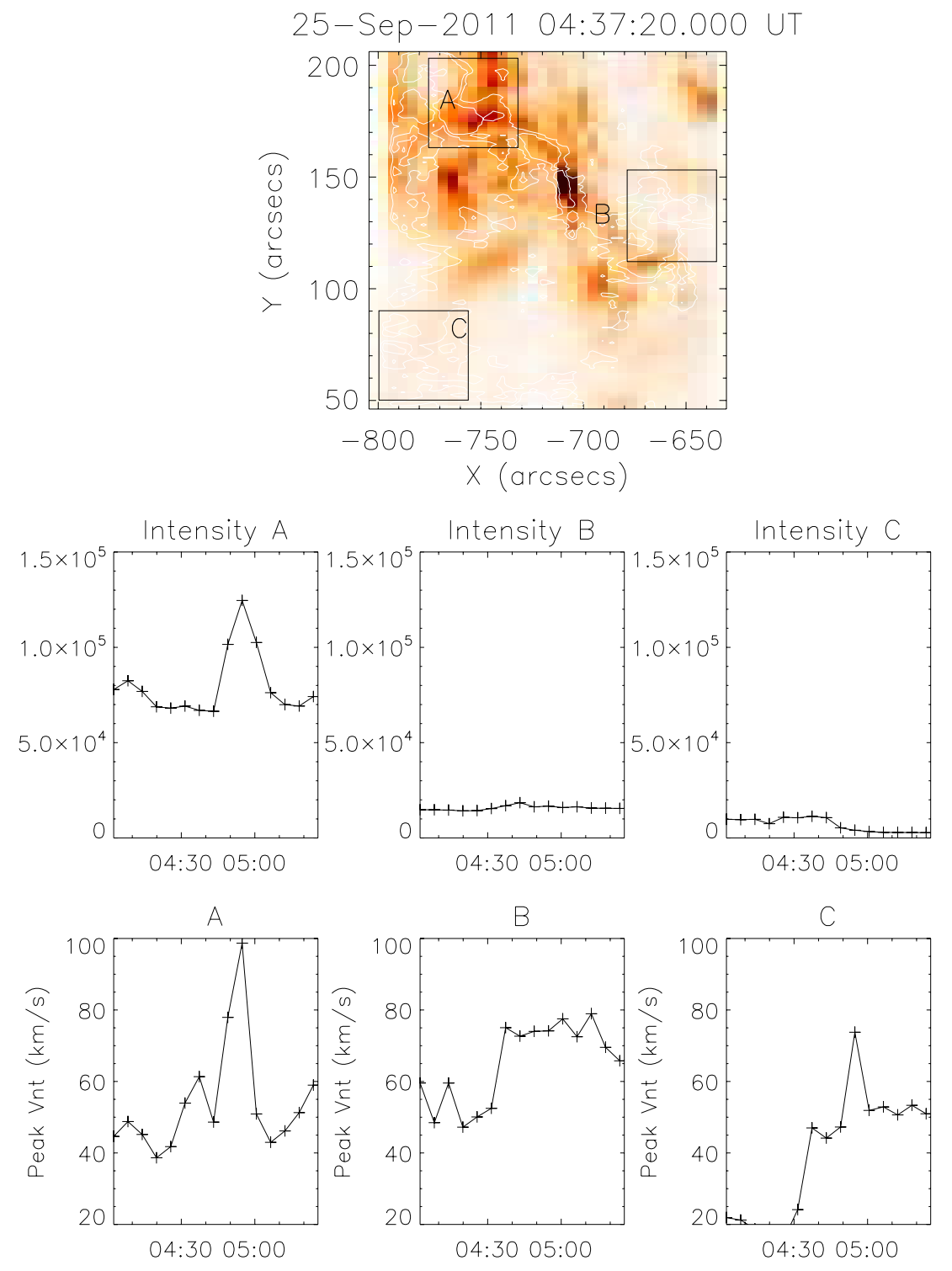

Figure 8. Top image shows the EIS intensity image of the Fe XII emission line at 15:32. Superimposed are three regions of interest. The contours are for the non-thermal velocity contours at levels 30 and $40 \mathrm{~km} \mathrm{~s}^{-1}$. The plots below the image show the intensity in these three regions and at the bottom the peak non-thermal velocity in each region. Region A shows the most dramatic changes in both intensity and Vnt. Region C also shows significant changes in the Vnt but little change in intensity.

(A color version of this figure is available in the online journal.)

with the HXR and non-thermal velocity contours overlaid. There is one HXR source that lies in the core of the active region. There is enhanced non-thermal velocity there, but in addition above and to the side of the bright loops. The temporal evolution of the non-thermal velocity is also shown (the active region before the event, the pre-flare, rise, and peak of the flare). At the pre-flare phase there are a number of pixels that are showing enhancements. One of the regions with enhancements is a distinct structure of roughly $20^{\prime \prime}$ in length. This structure lies along the region that is starting to erupt as seen in the AIA movies. In addition there is again a source to the west of the flaring region. The start of the flare shows strong enhancements that are in the core of the region and above, and at the peak of the flare the erupting structure can be clearly seen.

Figure 11 shows the time evolution of the Fe XII intensity and non-thermal velocity for the whole EIS field of view. In this case the intensity and non-thermal velocity rise at the same time and peak within the timescale of one EIS raster. The AIA images show the start, rise, and peak of the flare. At 15:26 UT a bright region is observed, which is highlighted by an arrow. This appears to be flare ribbons and is consistent with the early enhanced non-thermal velocity being in the brightest ribbon. By 15:32 UT the large eruption was underway. There is a weak CME related to this event, which has a linear speed of $440 \mathrm{~km} \mathrm{~s}^{-1}$.

Figure 12 shows lightcurves of the Fe XII intensity and nonthermal velocity for three regions in the EIS field of view. The highest intensity occurs in the main part of the active region. In the non-thermal velocity there are also two distinct peaks- the first one occurs early at 15:26 UT, and the second one occurs close in time to the intensity peak. Although region B is removed slightly from the core of the active region and shows no intensity peak, it shows significant fluctuations for well over an hour before the flare erupts. This region shows variations in all the previous flares described and is playing a role in the eruption of these events. Region C, which is located above the main bright looptops, shows an early small enhancement in intensity, but 

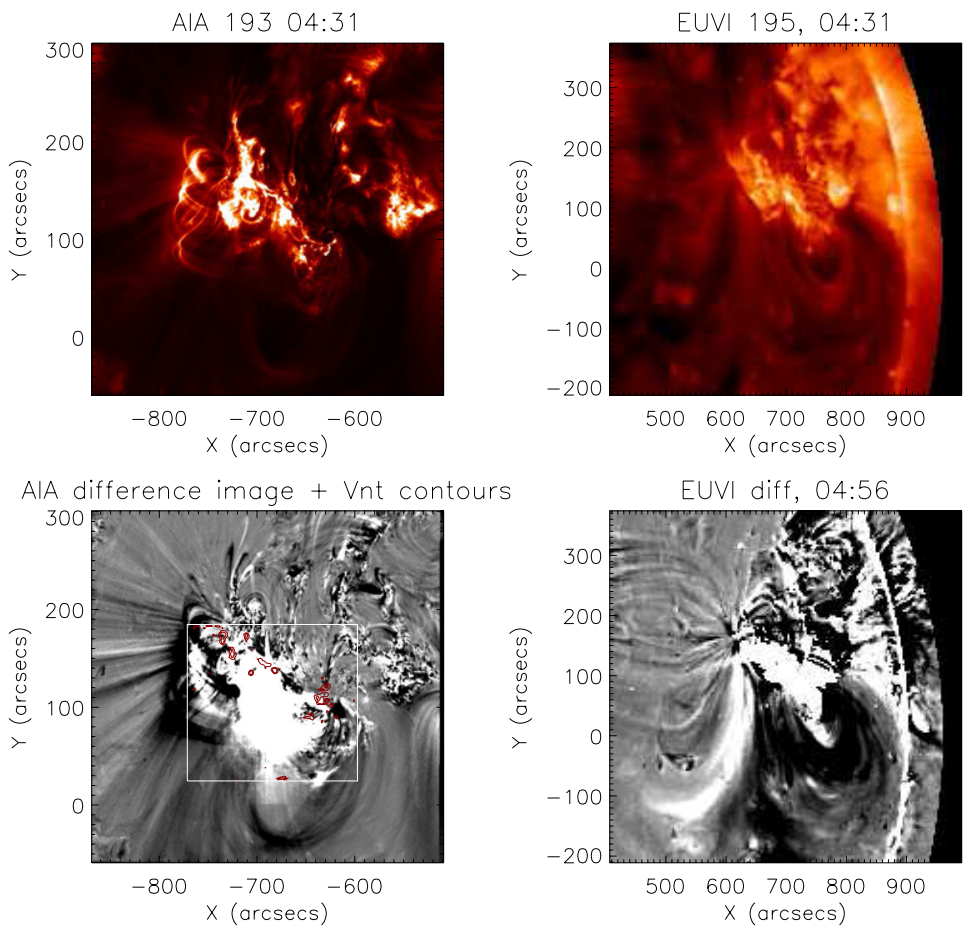

Figure 9. At top left is the AIA image taken before the flare began. On the lower left image, the AIA base difference image illustrates the dimming regions. The non-thermal velocity contours are shown in red (contour levels 40 and $50 \mathrm{~km} \mathrm{~s}^{-1}$ ) in the pre-flare phase. Contours showing enhanced non-thermal velocity lie along the erupting structure. To the west of the active region and at the end of the dimming footpoints there are also enhancements in the non-thermal velocity. The top right image shows the active region as viewed by STEREO B, before the flare occurred at September 24 04:31 At the bottom left the base difference image clearly shows the dimming region.

(A color version of this figure is available in the online journal.)

with no intensity enhancement at the flare peak. The intensity enhancement is related to a brightening of large-scale loops above the core of the active region. The non-thermal velocity shows a different story with three distinct peaks and high values being reached. The first peak is close in time to the peak of the intensity and there are two peaks later. Some caution needs to be used as there are strong blueshifts seen for the second nonthermal velocity peak in region A, so a secondary blueshifted component is required to the fit. The strong blueshifts are only seen in that one raster and are related to the observed eruption seen. The AIA animation shows smaller scale jets after the main eruption which may be contributing to the third small peak.

Figure 13 shows the AIA $193 \AA$ image and below the difference image with the non-thermal velocity contours overlaid. The STEREO-B EUVI $195 \AA$ Amages before the eruption and the difference image are also shown. The dimming region is again consistent with the two previous flares that occurred in the same active region. The western footpoint of the dimming region is consistent with the weak intensity region that shows large non-thermal velocity enhancements in the early phases.

\subsection{December 31 Flare}

This flare was designated an M2.4 GOES classification flare, and began at 13:09 UT on 2011 December 31. The flare occurred in NOAA active region number 1389 and was located S26E40 on the solar disk. This active region was flaring significantly from its appearance at the east limb, with a number of M-classification flares occurring before the one we analyze in detail. However, for at least $12 \mathrm{hr}$ before this flare occurred there was a steady, low background seen in GOES which was just above B-classification level. Figure 14 shows the AIA $193 \AA$ image with the contours of both the Fe XII non-thermal
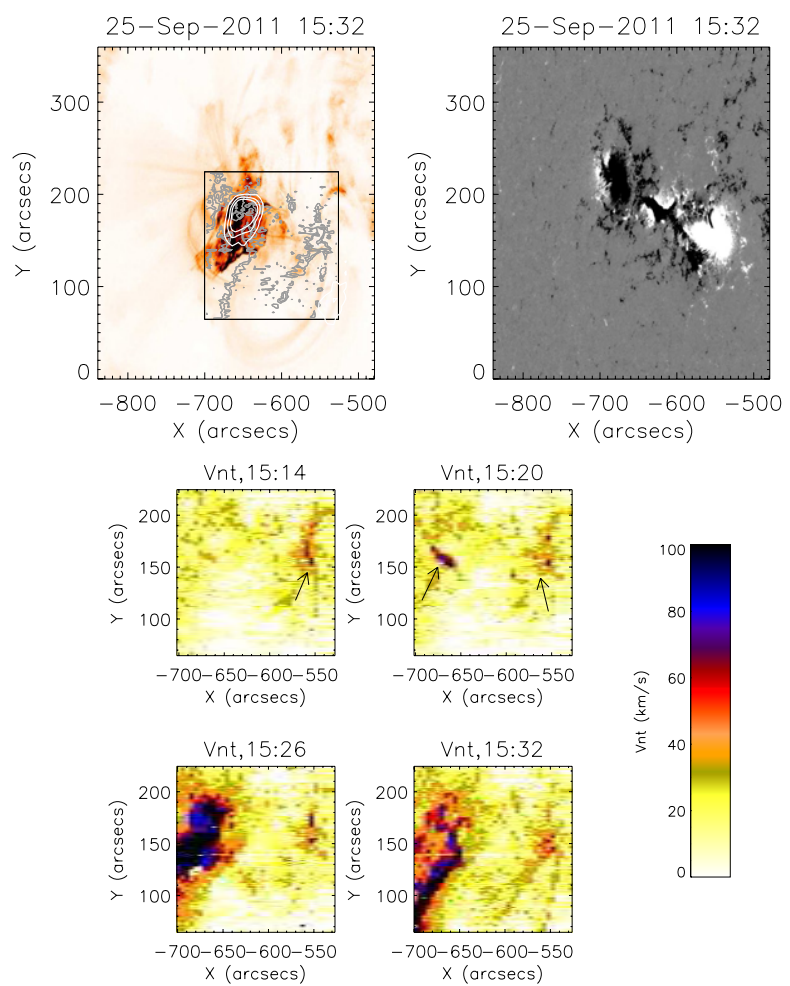

Figure 10. Top left panel shows an AIA 193 image at 09:29 with Vnt contours (Fe XII, $30-40 \mathrm{~km} \mathrm{~s}^{-1}$ ) in gray and the RHESSI contours in white $(12-25 \mathrm{keV})$. The box shows the location of the EIS field of view. The top right panel shows the line-of-sight magnetic field data from HMI. Below are four Vnt images taken at different times derived from Fe XII showing the location of the sources of high Vnt. The arrows in the second frame show the region which has a sudden enhancement in non-thermal velocity at the early stages of the event.

(A color version of this figure is available in the online journal.) 

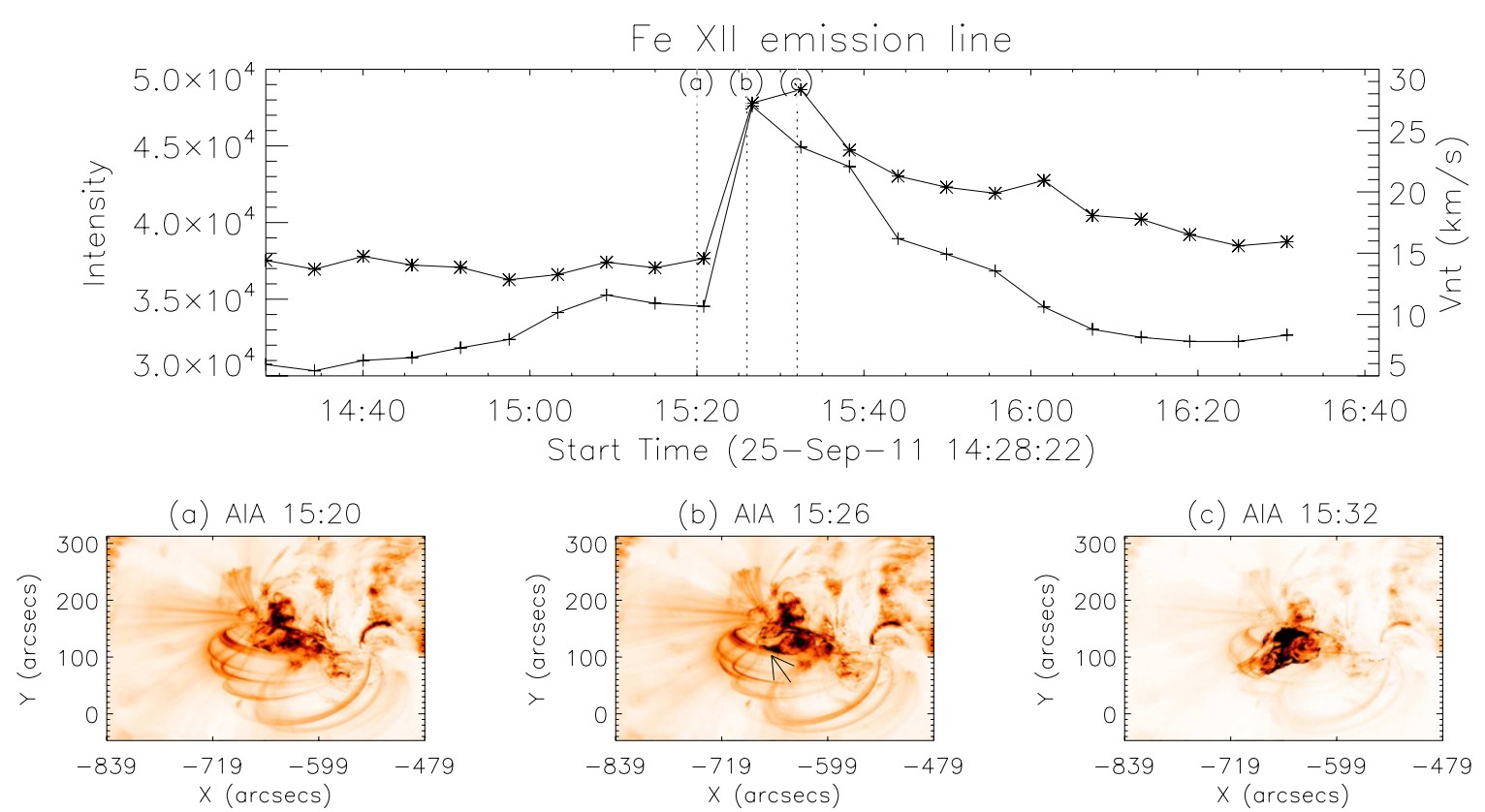

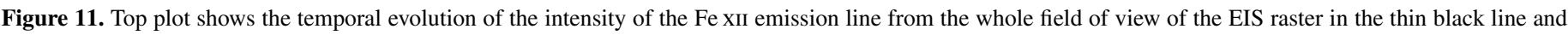

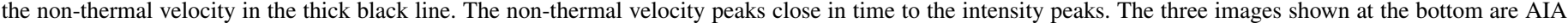

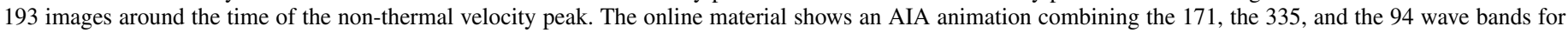
this flare. An intensity light curve of each waveband is also shown.

(An animation and color version of this figure are available in the online journal.)

velocity and the RHESSI contours at 3-6 keV. In addition four images of the Fe XII non-thermal velocity are shown at different times - the first time is representative of the active region with little activity, the second time is before any substantial increase in intensity as seen by EIS (or non-thermal velocity), the third time is close to the peak of the non-thermal velocity but the intensity is still low and the final image is around the peak of the intensity. In the EIS raster starting at 12:46 UT, there are a few pixels which are enhanced above the previous few rasters-one of the enhancements is highlighted by an arrow. These are located in the part of the active region that has smallscale brightenings seen in AIA which are shown in Figure 15. The HXR source derived from RHESSI is also compact and located in approximately the same location as the early brightenings and non-thermal velocity enhancement. The nonthermal velocity enhancement as the flare progresses stays reasonably compact covering an area of around $40^{\prime \prime} \times 40^{\prime \prime}$.

Figure 15 shows the lightcurves of the Fe XII intensity and non-thermal velocity derived from EIS. The temporal evolution of the intensity and non-thermal velocity is unusual. In this case there is a peak in non-thermal velocity which occurs related to a small intensity enhancement- - then there is a second nonthermal velocity peak which occurs at the same time as the intensity enhancement (the errors in the timings are within the time of an EIS raster, which is five minutes). AIA 193 $\AA$ images are shown in the pre-flare phase where small-scale enhancements of only a few arcseconds are seen. Close to the non-thermal velocity peak, there are small-scale intensity enhancements seen with no significant change in the overall magnetic morphology of the region. Both the RHESSI lightcurve and the GOES lightcurve peak at the same time as the first non-thermal velocity peak. It is somewhat unusual to have a situation when the EUV intensity increase is low compared to HXR and GOES. This may be related to the energy release being in such a confined region. By the time the intensity enhancement occurs, the active region has reconfigured and there are distinct loops around $50^{\prime \prime}$ in length. The GOES classification of the flare describes it as starting at 13:09 UT, and peaking at 13:15 UT. This is derived from full Sun data. Our light curves are derived from the EIS field of view, which is zoomed in on the small flaring region. The RHESSI lightcurve also behaves in a similar way to the GOES lightcurve.

Figure 16 shows the temporal variation of the Fe XII intensity and non-thermal velocity in three small regions of the active region. The dominant intensity enhancement is focused in region $B$, which also shows the small-scale intensity enhancements in AIA. The dominant non-thermal velocity enhancement occurs in the same region as the intensity enhancement but the peak occurs $\approx 40$ minutes earlier. Region $\mathrm{C}$ shows significant, but smaller in magnitude, enhancements in the non-thermal velocity at the same time as region B. The associated intensity enhancements occur much later. There is no CME associated with this flare.

\section{DISCUSSION}

In this work we have studied four flares. One was an unusual non-eruptive M-class flare, the other three events were from the same region, and they all had eruptions associated with the flares. The results we obtained were unexpected. They are summarized below:

1. Pre-event enhancement in non-thermal velocity at the base of the active region and its surroundings. There were preflare enhancements in all flares. These were in patches of a few pixels in size only in all cases. In the case of the noneruptive flare they occurred in the region that later flared. In the eruptive flares they occurred, in addition, away from the main flaring region, lying at the footpoints of the dimming region. 


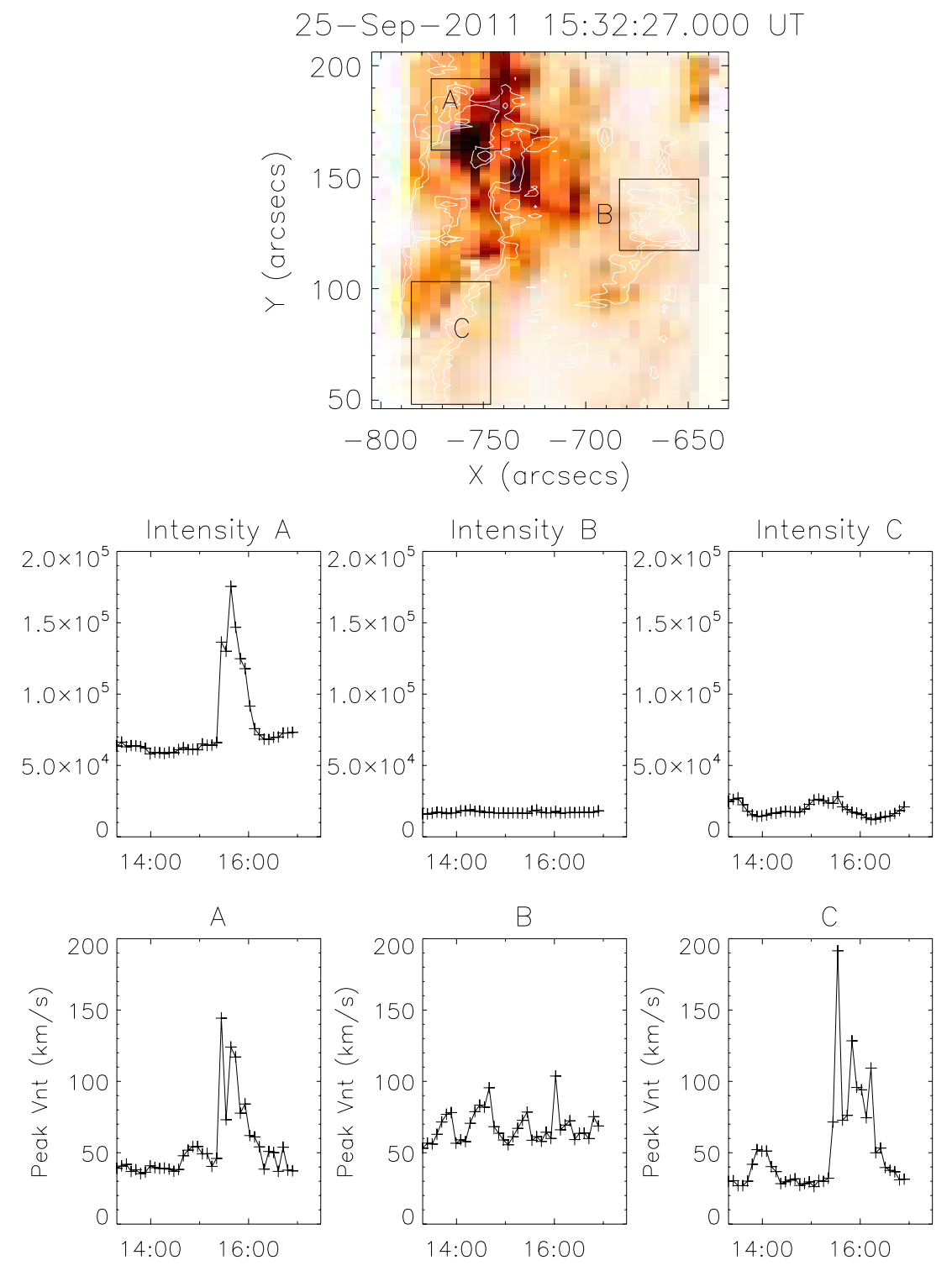

Figure 12. EIS intensity image of the Fe XII emission line at 15:32 is shown in the top panel. Superimposed are three regions of interest. The contours are of non-thermal velocity contours at levels 30 and $40 \mathrm{~km} \mathrm{~s}^{-1}$. The plots below the image show the intensity in these three regions and at the bottom the peak non-thermal velocity in each region. Region A shows the most dramatic changes in both intensity and Vnt. Region C also shows significant changes in Vnt but little change in intensity.

(A color version of this figure is available in the online journal.)

2. Pre-flare enhancements above the looptops. In all three eruptive flares there was evidence of non-thermal velocity enhancement close to or above the loop regions. These were consistent with the location of an untwisted structure in the September 24 flare, above the loops of the active region in September 25 04:30 flare, and at the leading edge of the erupting structure in the September 25 15:30 flare.

In all four cases the flare starts with small spatial scale changes seen in the non-thermal velocity. This is true for the non-eruptive flare as well for as the eruptive events.

The non-eruptive flare on the December 31 shows small-scale features that gradually brighten-these are likely to be small loop interactions. In the series of eruptive flares from active region NOAA 1302 in 2011 September, there are pre-flare nonthermal velocity enhancements seen in region A (part of the main flaring region) and region $\mathrm{B}$ (to the west of the flaring region with weak intensity) (see Figures 4, 8, and 12). The low intensity region $\mathrm{B}$ is clearly important in the triggering process.
When we look at the location of the dimming regions following the eruption it appears that the dimming region is homologous, located in approximately the same place for the three eruptive events. This deep dimming has one "footpoint" in the flaring part of the active region and one footpoint to the west of the main active region, which is where region $\mathrm{B}$ is. Coronal dimming is one of the best on-disk signatures of CMEs and was discovered during the Skylab era (Rust 1983). There have been suggestions that the dimmings are in fact consistent with the source of a CME being a flux rope (Sterling \& Hudson 1997). Spectroscopic observations of dimmings during the Hinode era have now confirmed that there is outflowing plasma associated with the dimmings and these outflowing regions can in fact be used to determine the magnetic flux involved in the eruption (Harra et al. 2011). In this paper, we have uncovered the activation of the region later associated with the dimming region.

One possible explanation for our observations is that we are seeing a coronal flux rope in the corona that is activated as the 

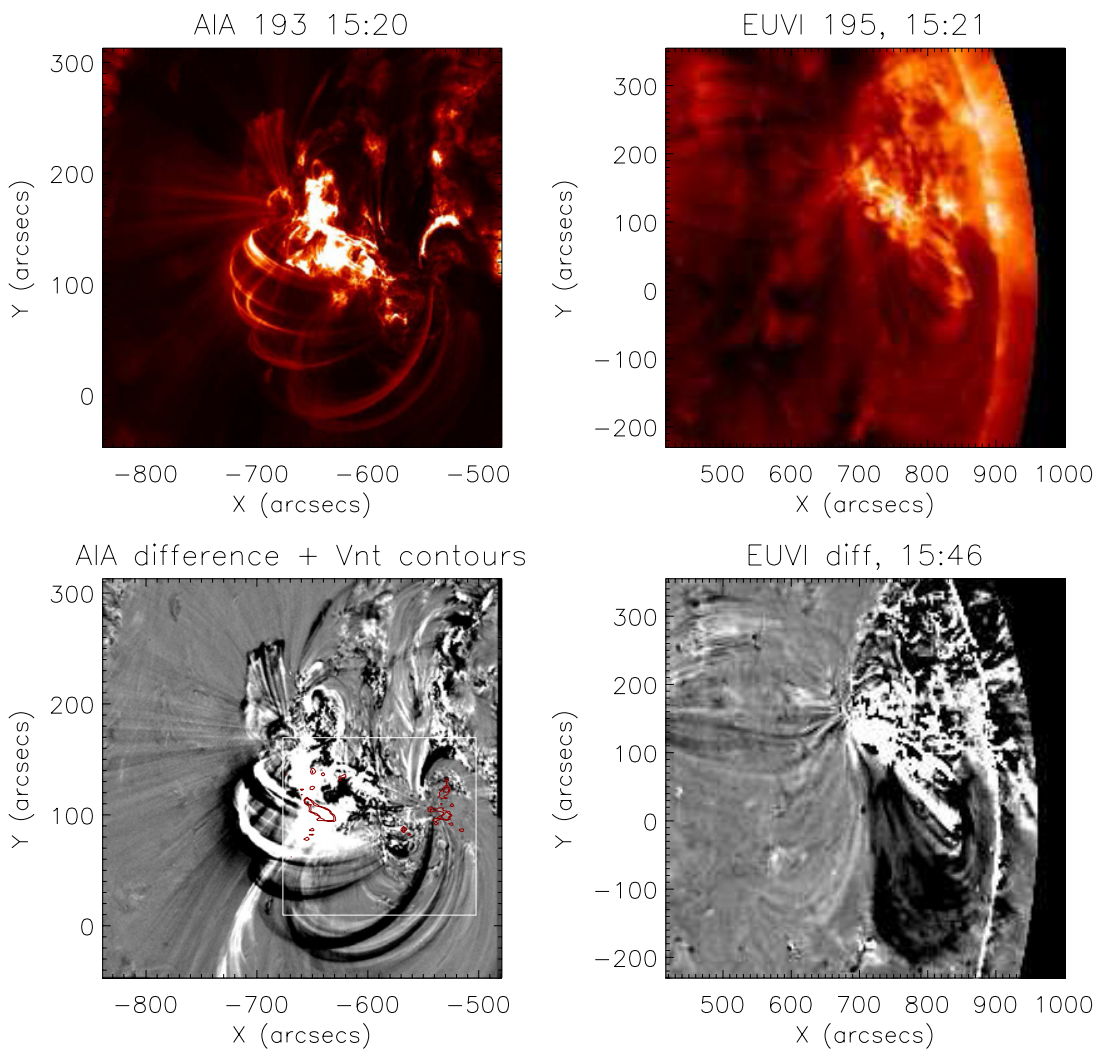

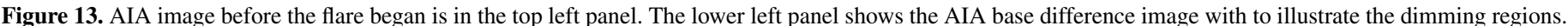

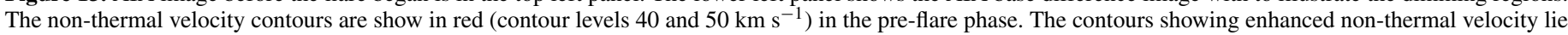

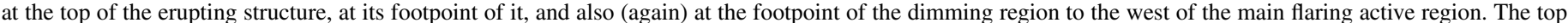

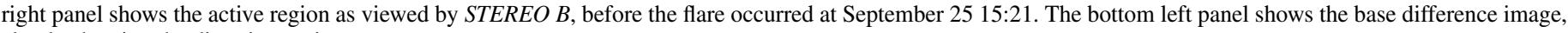
clearly showing the dimming region.

(A color version of this figure is available in the online journal.)

eruption begins. This has been a topic of considerable debate for many years - does the flux rope exist before CME onset or is it formed during the eruption itself, e.g., Forbes (2000)? A lot of work has been done on flux ropes that emerge from quiet Sun regions, but in the case of active regions it is more complex due to the difference in intensity, dynamics and size scale (a flux rope is likely to be much smaller in an active region). New results have appeared that made use of the higher spatial and temporal resolution and broader temperature range images available from SDO-AIA. Patsourakos et al. (2013) have found evidence of a formation of a coronal magnetic flux rope during a confined flare that then erupted $7 \mathrm{hr}$ later. Cheng et al. (2011, 2013) found that the flux rope first appears as a twisted hot feature that lies along the polarity inversion line and it plays an important initiation role in the CME. Explanations for the appearance of flux ropes before their eruption have been given by Green et al. (2011) showing that flux cancelation is an important process in the creation of a flux rope. In their case around one-third of the active region flux cancels at the polarity inversion line 2.5 days before the eruption occurred which leads to the formation of a sheared coronal structure. Savcheva et al. (2012) carried out MHD simulations to understand the formation and evolution of highly sheared sigmoidal structures - they also find that flux cancelation plays an important role in creating the flux rope.

There are other explanations for the existence of small spatial non-thermal velocity enhancements remote from the flare site. Kusano et al. (2012) have carried out three-dimensional MHD simulations to probe whether small-scale flux emergence of the opposite polarity could also trigger a flare. They found that it is possible that if small-scale new flux with the opposite polarity to the potential component emerges near the polarity inversion line that this is enough to trigger a flare. Detailed numerical modeling (either by field extrapolation or data-driven modeling) of AR 11302 will be needed to shed light on the relevant physical mechanisms that facilitate multiple eruptions from this particular active region. However, the morphology of the photospheric field in AR 11302 certainly has properties that are associated with flares and CME-productive regions. For instance, the magnetogram shown in, for example, Figure 2 reveals a quadrapolar morphology, which is amenable to flux rope ejections from a breakout scenario (Antiochos et al. 1994). Evidence has been found to support the breakout model by Sterling \& Moore (2001) who observed remote brightenings suggestive of reconnection occurring to release the flux rope. In addition, the sharp polarity inversion line and the apparent shearing motion about the polarity inversion line is a suitable location for energy input into the AR. In their data-driven simulation of AR 11158 (another active region with multiple eruptions), Cheung \& DeRosa (2012) found that a systematic shearing motion at the photospheric boundary led to the ejection of a series of flux ropes (see also Manchester et al. 2004 for the possible subsurface origin of shearing motions).

Regardless of the physical mechanisms that lead to eruptions and flares, what is clear from this work is the presence of pre-flare enhancements in non-thermal velocity both in the flare site and remote from it. Regardless of the pre-existing coronal magnetic configuration or the triggering mechanism for eruption, this work suggests that plasma motions (seen in 

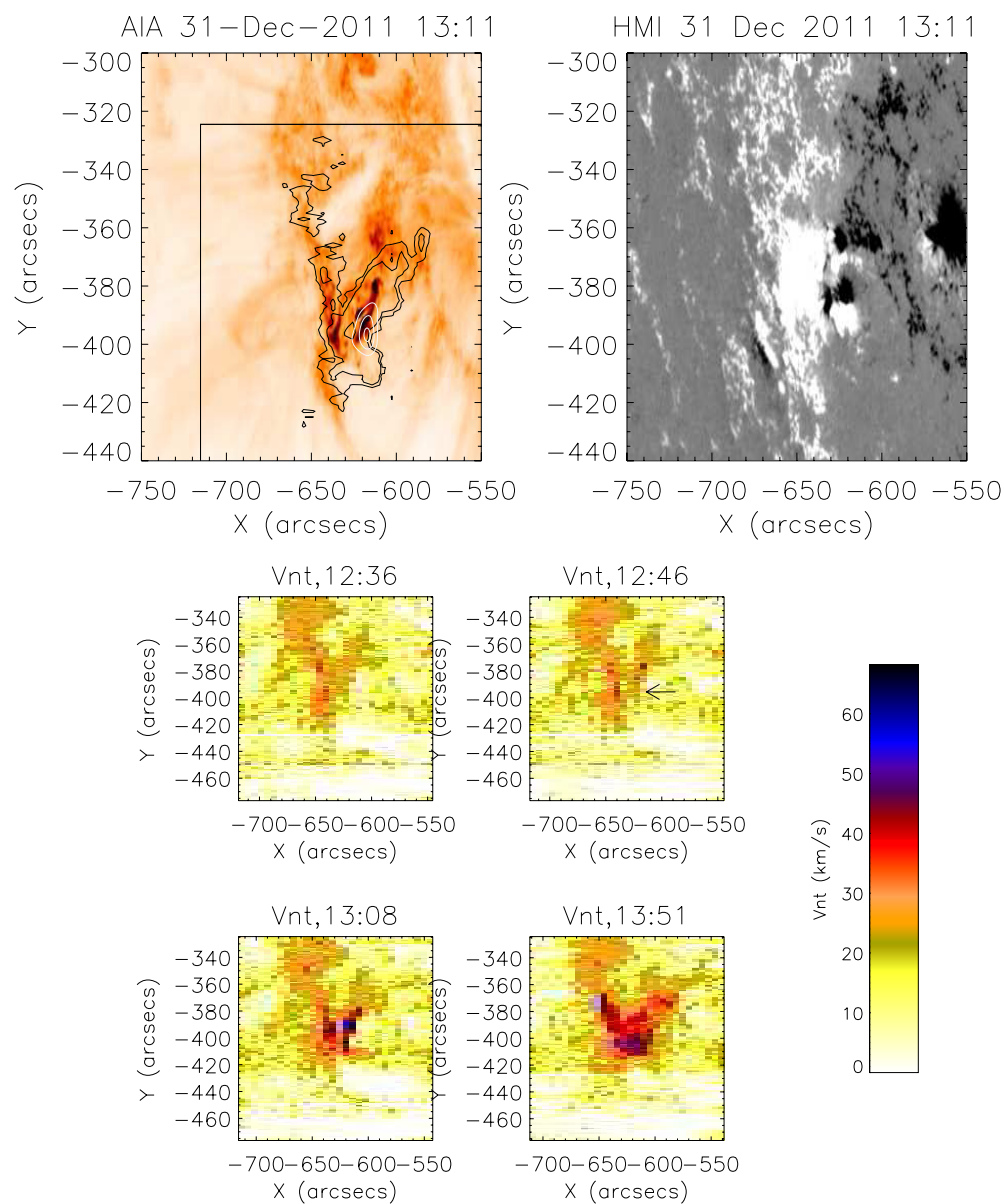

Figure 14. Top left panel shows an AIA 193 image at 13:11 with Vnt contours (Fe XII, 30-40 $\mathrm{km} \mathrm{s}^{-1}$ ) in black and the RHESSI contours in white (3-6 keV). The box shows the location of the EIS field of view. The top right panel shows the line-of-sight magnetic field data from HMI. Below are four Vnt images at different times derived from Fe XII showing the location of the sources of high Vnt. The arrow in the non-thermal velocity image at 12:46 UT highlights one of the small pre-event enhancement in non-thermal velocity.

(A color version of this figure is available in the online journal.)

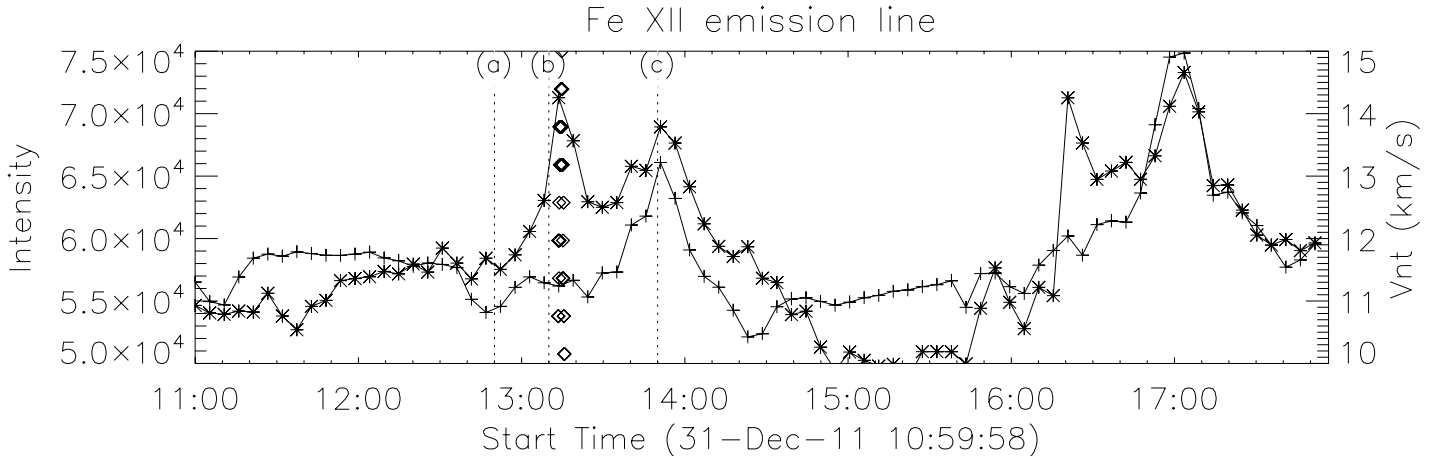

(a) AIA 12:50

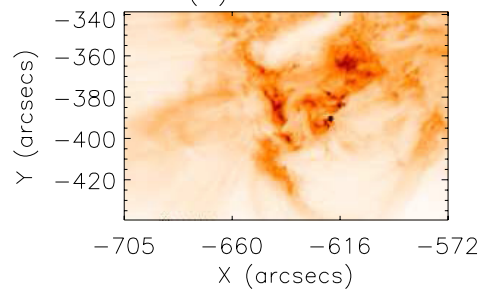

(b) AIA 13:10

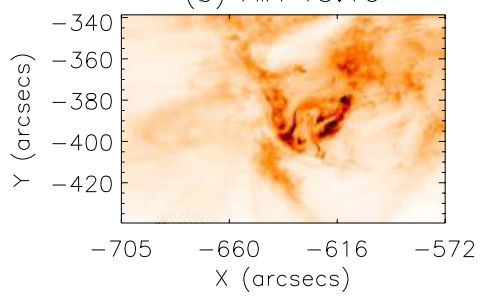

(c) AIA 13:50

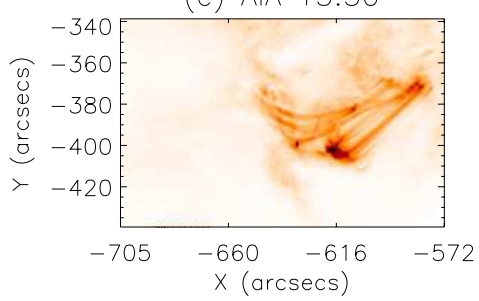

Figure 15. Top plot shows the Fe XII emission line intensity from the whole field of view of the EIS raster (thin black line) and the non-thermal velocity (thick black line). The non-thermal velocity peaks nearly 40 minutes before the peak intensity. The RHESSI lightcurve for the 6-12 keV energy range is shown with diamonds, peaking with the first non-thermal velocity peak. The three panels shown at the bottom are AIA 193 images around the time of the first non-thermal velocity peak, showing the small scales change that are occurring at that time. The online material shows an AIA animation combining the 171, the 211, and the 94 wave bands for this flare. An intensity light curve of each waveband is also shown.

(An animation and color version of this figure are available in the online journal.) 


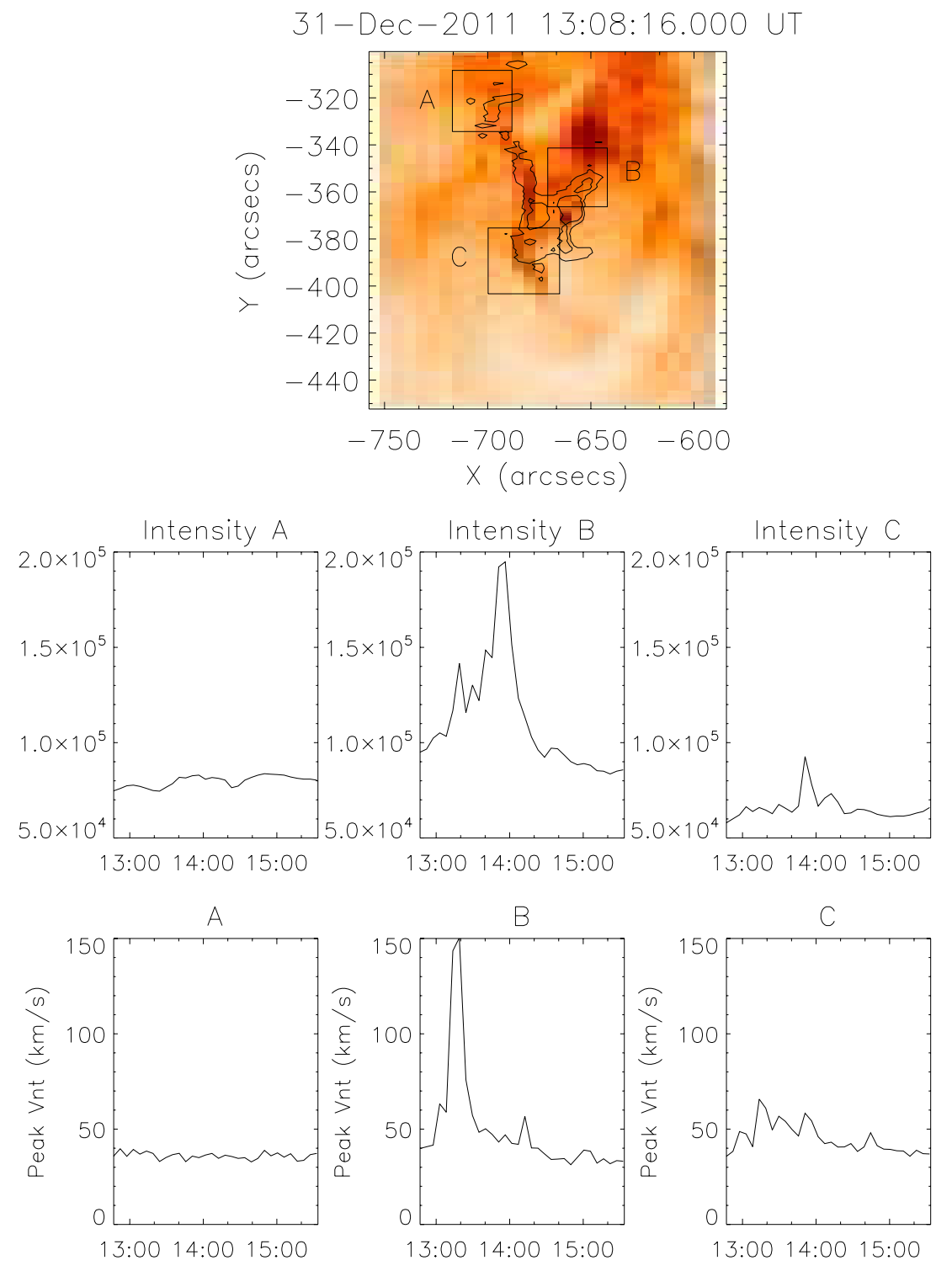

Figure 16. Top panel shows the Fe XII emission line intensity image at 13:08 UT. Superimposed are three regions of interest. The contours are for non-thermal velocity contours at levels 30 and $40 \mathrm{~km} \mathrm{~s}^{-1}$. The panels below the image show the intensity in these three regions; the bottom panels show the peak non-thermal velocity in each region. Region B shows the most dramatic changes with the peak in the Vnt occurring nearly 40 minutes before the peak in the intensity.

(A color version of this figure is available in the online journal.)

imaging data from AIA) associated with the initial phases of an eruption (i.e., before the onset of the resulting flare) are likely responsible for the enhanced non-thermal velocities detected in coronal emission lines. In the three eruptive flares we studied, all show enhancement in the same locations, suggesting the eruptions are homologous in nature and that whatever is erupting re-forms to start over again.

We are grateful to ISSI for the support of the emerging flux team led by Klaus Galsgaard. Hinode is a Japanese mission developed and launched by ISAS/JAXA, collaborating with NAOJ as a domestic partner, and NASA and STFC (UK) as international partners. Scientific operation of the Hinode mission is conducted by the Hinode science team organized at ISAS/ JAXA. This team mainly consists of scientists from institutes in the partner countries. Support for the post-launch operation is provided by JAXA and NAOJ (Japan), STFC (UK), NASA, ESA, and NSC (Norway). Courtesy of NASA/SDO and the
AIA, EVE, and HMI science teams. M.C.M.C. acknowledges support from NASA's AIA contract NNG04EA00C to LMSAL. The research leading to these results has received funding from the European Commission's Seventh Framework Programme under the grant agreement No. 284461 (eHEROES project).

\section{REFERENCES}

Antiochos, S. K., Dahlburg, R. B., \& Klimchuk, J. A. 1994, ApJL, 420, L41 Cheng, X., Zhang, J., Ding, M. D., Liu, Y., \& Poomvises, W. 2013, ApJ, 763,43

Cheng, X., Zhang, J., Liu, Y., \& Ding, M. D. 2011, ApJL, 732, L25

Cheung, M. C. M., \& DeRosa, M. L. 2012, ApJ, 757, 147

Culhane, J. L., Harra, L. K., James, A. M., et al. 2007, SoPh, 243, 19

Doschek, G. A., Feldman, U., Kreplin, R. W., \& Cohen, L. 1980, ApJ, 239, 725

Forbes, T. G. 2000, JGR, 105, 23153

Green, L. M., Kliem, B., \& Wallace, A. J. 2011, A\&A, 526, A2

Grigis, P., Su, Y., \& Weber, M. 2013, SDO document (LMSAL), http://www.lmsal.com/sdodocs/doc?cmd=dcur\&proj_num=SDOD0076\& file_type $=$ pdf 
Hara, H., Watanabe, T., Harra, L. K., Culhane, J. L., \& Young, P. R. 2011, ApJ, 741, 107

Harra, L. K., Mandrini, C. H., Dasso, S., et al. 2011, SoPh, 268, 213

Harra, L. K., Matthews, S. A., \& Culhane, J. L. 2001, ApJL, 549, L245

Harra, L. K., Williams, D. R., Wallace, A. J., et al. 2009, ApJL, 691, L99

Hassler, D. M., Rottman, G. J., Shoub, E. C., \& Holzer, T. E. 1990, ApJL, 348, L77

Inoue, S., Shiota, D., Yamamoto, T. T., et al. 2012, ApJ, 760, 17

Kontar, E. P., Hannah, I. G., \& Bian, N. H. 2011, ApJL, 730, L22

Kosugi, T., Matsuzaki, K., Sakao, T., et al. 2007, SoPh, 243, 3

Kurokawa, H., Wang, T., \& Ishii, T. T. 2002, ApJ, 572, 598

Kusano, K., Bamba, Y., Yamamoto, T. T., et al. 2012, ApJ, 760, 31

Lemen, J. R., Title, A. M., Akin, D. J., et al. 2012, SoPh, 275, 17

Lin, R. P., Dennis, B. R., Hurford, G. J., et al. 2002, SoPh, 210, 3

Lucy, L. B. 1974, AJ, 79, 745

Magara, T., \& Tsuneta, S. 2008, PASJ, 60, 1181

Manchester, W., IV, Gombosi, T., DeZeeuw, D., \& Fan, Y. 2004, ApJ, 610, 588
Milligan, R. O. 2011, ApJ, 740, 70

Patsourakos, S., Vourlidas, A., \& Stenborg, G. 2013, ApJ, 764, 125

Richardson, W. H. 1972, JOSA, 62, 55

Rust, D. M. 1983, SSRv, 34, 21

Savcheva, A., Pariat, E., van Ballegooijen, A., Aulanier, G., \& DeLuca, E. 2012, ApJ, 750, 15

Scherrer, P. H., Schou, J., Bush, R. I., et al. 2012, SoPh, 275, 207

Sterling, A. C., \& Hudson, H. S. 1997, ApJL, 491, L55

Sterling, A. C., \& Moore, R. L. 2001, ApJ, 560, 1045

Wallace, A. J., Harra, L. K., van Driel-Gesztelyi, L., Green, L. M., \& Matthews, S. A. 2010 , SoPh, 267,361

Warren, H. P., \& Warshall, A. D. 2001, ApJL, 560, L87

Watanabe, T., Hara, H., Sterling, A. C., \& Harra, L. K. 2012, SoPh, 281, 87

Williams, D. R., Harra, L. K., Brooks, D. H., Imada, S., \& Hansteen, V. H. 2009, PASJ, 61, 493

Wuelser, J.-P., Lemen, J. R., Tarbell, T. D., et al. 2004, Proc. SPIE, 5171, 111

Yashiro, S., Gopalswamy, N., Michalek, G., et al. 2004, JGRA, 109, 7105 